\title{
Association of Arthrogryposis Multiplex Congenita with Maternal Antibodies Inhibiting Fetal Acetylcholine Receptor Function
}

\author{
Sietske Riemersma, ${ }^{*}$ Angela Vincent, ${ }^{\star}$ David Beeson, ${ }^{\star}$ Claire Newland, ${ }^{\star}$ Simon Hawke, ${ }^{\star}$ Beatrice Vernet-der Garabedian, ${ }^{\ddagger}$ \\ Bruno Eymard, ${ }^{\ddagger}$ and John Newsom-Davis* \\ *Neurosciences Group, Department of Clinical Neurology, University of Oxford, Institute of Molecular Medicine, John Radcliffe \\ Hospital, Oxford OX3 9DU, United Kingdom; and ${ }^{\ddagger}$ Institut Nationale de la Santé et de la Recherche Médicale U 25, Hôpital Necker, \\ Paris, France
}

\begin{abstract}
Arthrogryposis multiplex congenita (AMC), characterized by multiple joint contractures developing in utero, results from lack of fetal movement. Some cases are genetically determined, but AMC occasionally complicates pregnancy in patients with myasthenia gravis (MG) suggesting involvement of circulating maternal antibodies. We previously demonstrated antibodies that inhibited the function of fetal acetylcholine receptor (AChR) in one healthy woman with an obstetric history of recurrent AMC. Here we study sera from this woman, from one other with a similar history, and from three (one asymptomatic) whose babies had neonatal MG and AMC. All five maternal sera had high titers of antibodies that inhibited $\alpha$-Bungarotoxin ( $\alpha$-BuTx) binding to fetal AChR, and their sera markedly inhibited fetal AChR function with little effect on adult AChR function. Moreover, in a further survey, 3 of 20 sera from anti-AChR negative AMC mothers inhibited fetal AChR function significantly at 1:100 dilution. These results demonstrate the role of antibodies to fetal AChR and perhaps other muscle antigens in some cases of AMC. More generally, they suggest that placental transfer of antibodies directed at fetal antigens should be considered as a cause of other recurrent fetal or perinatal disorders. (J. Clin. Invest. 1996. 98:2358-2363.) Key words: myasthenia gravis $\bullet$ arthrogryposis $\bullet$ acetylcholine receptor $\bullet$ placental-to-fetal transfer $\bullet$ autoantibody
\end{abstract}

\section{Introduction}

Arthrogryposis multiplex congenita (AMC) $)^{1}$ is characterized by the presence of multiple joint contractures developing in

Address correspondence to Dr. Angela Vincent, Neurosciences Group, Institute of Molecular Medicine, John Radcliffe Hospital, Oxford OX3 9DU. Phone: 44-1865-222323; FAX: 44-1865-222402; E-mail: neurosciences@imm.ox.ac.uk

Received for publication 5 June 1996 and accepted in revised form 23 September 1996.

1. Abbreviations used in this paper: $\mathrm{ACh}$, acetylcholine; $\mathrm{AChR}$, acetylcholine receptor; AMC, arthrogryposis multiplex congenita; AMC-M, mother of AMC offspring; $\alpha$-BuTx, $\alpha$-bungarotoxin; MG, myasthenia gravis.

J. Clin. Invest.

(C) The American Society for Clinical Investigation, Inc. 0021-9738/96/11/2358/06 \$2.00

Volume 98, Number 10, November 1996, 2358-2363 utero. In most cases it results from lack of fetal movement that can be associated with intrauterine or neonatal death due to pulmonary hypoplasia and hydramnios. Some cases are genetically determined but in others the cause is unknown (1). Chick embryos injected with the muscle acetylcholine receptor (AChR) blocking drug, $d$-tubocurarine, developed joint contractures (2), and AMC occurred in the baby of a mother with tetanus who was receiving muscle relaxants (3). Thus, maternal circulating factors inhibiting muscle activity need to be considered as possible causes of AMC.

Myasthenia gravis (MG) is an autoimmune condition in which serum antibodies to AChR lead to loss of AChR and muscle weakness (4). The pentameric AChR exists in two forms; $\alpha_{2} \beta \gamma \delta$ in fetal or denervated muscle, and $\alpha_{2} \beta \delta \epsilon$ in adult muscle (5). A proportion of babies born to MG mothers exhibit transient weakness (neonatal MG) (6), and this is more frequent if the mother's antibody binds preferentially to fetal AChR (7). Occasionally babies have AMC as well as neonatal MG $(6,7)$, suggesting that movement had been severely impaired in utero.

We have recently reported the case of a woman whose first healthy child was followed by five pregnancies complicated by severe fetal AMC leading to stillbirth or termination of pregnancy (8). Although at no time having symptoms of $\mathrm{MG}$, and showing no clinical features of MG when examined at the time of her most recent pregnancy, she had high levels of antiAChR and her immunoglobulin G (IgG) specifically inhibited the function of fetal, but not adult, AChR expressed in Xenopus oocytes (8). Another patient, in whom MG was eventually diagnosed, had high titers of anti-AChR antibodies and a similar obstetric history (9). In this paper we define the specificity of anti-AChR antibodies in sera from these two women and from three other mothers whose babies were born with AMC and who had formed part of an earlier survey of neonatal $\mathrm{MG}$ (7). The latter three were also positive for anti-AChR but one was asymptomatic. We show that antibodies inhibiting fetal $\mathrm{AChR}$ function are present at high concentration in all five sera, confirming the importance of this antibody specificity in causing some cases of AMC. This work was reported in abstract (10).

\section{Methods}

Patient sera. Sera were obtained from five mothers (M) with obstetric histories of $\mathrm{AMC}$, who were known to be anti-AChR antibody positive, as described elsewhere; AMC-M1 (8), AMC-M2 (9), AMCM3-5 $(6,7)$. The main clinical features are summarized in Table I. In addition, we screened sera from 20 mothers with obstetric histories of AMC, who had no clinical features of MG or any other known immunological association. These sera were sent by clinical geneticists. 
Table I. Summary of AMC Mothers (AMC-M) and Their Obstetric Histories

\begin{tabular}{lcccccc}
\hline & & & \multicolumn{5}{c}{ Pregnancies } \\
\cline { 5 - 7 } & & $\begin{array}{c}\text { Age } \\
\text { at sampling }\end{array}$ & $\begin{array}{c}\text { Clinical } \\
\text { signs of MG }\end{array}$ & $\begin{array}{c}\text { Stillborn } \\
\text { Total }\end{array}$ & $\begin{array}{c}\text { Neonatal } \\
\text { or terminated }\end{array}$ & $\begin{array}{c}\text { Surviving } \\
\text { MG/AMC } \\
\text { offspring }\end{array}$ \\
\hline AMC-M1 & 32 & - & 6 & 5 & 0 & 1 (1st) \\
AMC-M2 & 29 & + & 4 & 3 & 1 & 1 (2nd) \\
AMC-M3 & 35 & + & 6 & 5 & 1 & 1 (6th) \\
AMC-M4 & 30 & + & 1 & 0 & 1 & 1 (1st) \\
AMC-M5 & 28 & - & 2 & 1 & 1 & 1 (2nd)
\end{tabular}

Measurement of anti-AChR antibodies. Anti-AChR was measured by conventional radioimmunoassay using ${ }^{125} \mathrm{I}-\alpha$-BuTx $(>200$ $\mathrm{mCi} / \mathrm{mmol}$, Amersham International, Little Chalfont, UK) to label human muscle AChR extracted from a mixture of the ischemic muscles of amputated legs (11). The AChR preparation contained mostly fetal (denervated) type AChR (as shown by reactivity with monoclonal antibodies specific for fetal AChR [12]) with a small amount of adult-type AChR. $50 \mu \mathrm{l}$ of prelabeled muscle extract was incubated with serum dilutions for $2 \mathrm{~h}$ at room temperature, and complexes precipitated with excess goat anti-human Ig using normal human serum as carrier if necessary. Results were expressed as nanomoles of $\alpha$-BuTx binding sites precipitated per liter of serum, using data from the linear part of the binding curve.

Inhibition of ${ }^{125} I-\alpha-B u T x$ binding. Antibodies directed towards the ${ }^{125} \mathrm{I}-\alpha-\mathrm{BuTx}$ binding sites were measured by preincubating $50 \mu \mathrm{l}$ of the muscle extract with different dilutions of the patient's sera in $50 \mu \mathrm{l}$ of PTX buffer for $2 \mathrm{~h}$ at room temperature before adding ${ }^{125} \mathrm{I}-\alpha-$ BuTx at saturating concentrations $(2-3 \mathrm{nM}) .{ }^{125} \mathrm{I}-\alpha-\mathrm{BuTx}-\mathrm{AChR}$ complexes were precipitated as above by addition of $1 \mu \mathrm{l}$ of a MG serum pool, to bind AChR, followed by excess goat anti-human IgG. Results were expressed as nanomoles of binding sites inhibited/liter of serum, based on the amount of ${ }^{125} \mathrm{I}-\alpha-\mathrm{BuTx}$ binding inhibited by $0.16 \mu$ l of each AMC-M serum.

${ }^{22} \mathrm{Na}^{+}$flux assay. AChR function in TE671 cells was measured as previously described $(13,14)$. TE671 cells (here called TE671- $\gamma$ ) that express fetal AChR, or TE671- $\epsilon$, a subline in which over $70 \%$ of the AChR is the adult form (15), were grown under standard conditions and plated at $1-2 \times 10^{6}$ cells per $32 \mathrm{~mm}$ well. $2 \mathrm{~d}$ later the medium was removed and replaced with serum or $\operatorname{IgG}$ dilutions in Hepes Locke buffer for $1 \mathrm{~h}$ at room temperature. The cells were briefly washed and the ${ }^{22} \mathrm{Na}^{+}$flux measured using 1 min application of $0.2-$ $0.5 \mathrm{mM}$ carbachol as agonist and $300,000 \mathrm{cpm} / \mathrm{ml}$ of ${ }^{22} \mathrm{Na}^{+}$in Hepes Locke buffer. The wells were washed rapidly four times with $2 \mathrm{ml}$ of Hepes Locke buffer, and the retained ${ }^{22} \mathrm{Na}^{+}$counted on a gamma counter. Results were compared with wells incubated in buffer alone, after subtraction of cpm in wells pretreated with excess $\alpha$-BuTx to inhibit AChR function. In some experiments serum was tested similarly after a 30-min preincubation in $\mathrm{mAbs}$ to $\mathrm{AChR}$ (ascites diluted at 1:250-1:500; [12]). Based on preliminary experiments, this concentration was sufficient to bind all the surface AChR.

Effects of IgG on fetal and adult AChR expressed in Xenopus oocytes. IgG was purified using protein A sepharose under standard conditions using a Pierce kit. IgG fractions were dialyzed overnight against Ringer's solution. Expression of human AChR in Xenopus oocytes and measurement of ACh-induced ion currents was performed as described $(8,16)$. Briefly, oocytes were injected with cRNAs encoding either fetal $\left(\alpha_{2} \beta \gamma \delta\right)$ or adult $\left(\alpha_{2} \beta \delta \epsilon\right)$ type human AChR subunits. ACh-induced currents were measured by conventional two microelectrode voltage clamp 2-7 d following injection. Currents evoked by $1 \mathrm{mM}$ ACh were measured before and immediately after a 40-min incubation in IgG at 1:50 dilution.

\section{Results}

Anti-AChR antibodies in AMC-mothers. We first examined serum anti-AChR antibodies by immunoprecipitation. All AMC-M sera precipitated ${ }^{125} \mathrm{I}-\alpha-\mathrm{BuTx}-\mathrm{AChR}$ at high dilution (Fig. $1 A$ ). In titrations of sera from AMC-M1, AMC-M2, and AMC-M4, however, only about $40-50 \%$ of the ${ }^{125} \mathrm{I}-\alpha-\mathrm{BuTx}$ was precipitated even with $4 \mu \mathrm{l}$ of each serum, compared with that precipitated when AMC-M3 and AMC-M5 sera (Fig. 1 $A$ ), or a serum pool from typical MG patients (not shown) were tested. This suggested, from previous evidence (17), that these three AMC-M sera were partially displacing ${ }^{125} \mathrm{I}-\alpha-\mathrm{BuTx}$ from its binding sites on the AChR. We therefore looked directly for antibodies against the $\alpha$-BuTx binding sites. When



B



Figure 1. (A) Immunoprecipitation of AChR from ischemic human leg muscle containing mainly fetal-type AChR. AMC-M1 (ם), AMCM2 ( $\mathbf{\Lambda})$, and AMC-M4 ( ) precipitate only about $40-50 \%$ of the available ${ }^{125} \mathrm{I}-\alpha-\mathrm{BuTx}$ binding sites, in contrast to AMC-M3 ( $\left.\boldsymbol{\nabla}\right)$ and AMC-M5 (-) that precipitate all available binding sites. $(B)$ Inhibition of ${ }^{125} \mathrm{I}-\alpha-$ BuTx binding to ischemic leg muscle AChR by preincubating in AMC-M sera. All the sera strongly inhibit binding of ${ }^{125} \mathrm{I}-$ $\alpha$-BuTx. An MG serum pool has little effect ( $\square$ ). Some of the data points have been omitted for clarity. 
Table II. Anti-AChR in AMC Mothers

\begin{tabular}{|c|c|c|c|c|}
\hline & \multirow{2}{*}{$\frac{\text { Anti-AChR }}{n M}$} & \multirow{2}{*}{$\begin{array}{c}\begin{array}{c}\text { Anti- } \alpha-\mathrm{BuTx} \\
\text { site }\end{array} \\
n M\end{array}$} & \multicolumn{2}{|c|}{$\begin{array}{l}\% \text { inhibition of } \\
{ }^{22} \mathrm{Na}^{+} \text {flux } \\
(1: 100 \text { dilution })^{* \neq}\end{array}$} \\
\hline & & & $T E 671-\gamma$ & TE671- $\epsilon$ \\
\hline AMC-M1 & 62 & 96 & 99 & -4 \\
\hline AMC-M2 & 421 & 140 & 94 & 3 \\
\hline AMC-M3 & $>3000$ & 131 & 90 & 4 \\
\hline AMC-M4 & 68 & 132 & 87 & 7 \\
\hline AMC-M5 & $>3000$ & 86 & 88 & 16 \\
\hline Typical MG patients & $0-10,000$ & $0-10$ & $0-60$ & $0-45$ \\
\hline Healthy & $<0.2$ & $<0.5$ & $0-20$ & nd \\
\hline
\end{tabular}

*Very similar results were obtained at 1:25 dilution. ${ }^{\ddagger}$ Insufficient serum was available from AMC-M3-5 to perform full titrations. TE671- $\gamma$ and TE761- $\epsilon$ cells showed similar inhibition of ${ }^{22} \mathrm{Na}^{+}$flux $(>85 \%)$ by $d$-tubocurarine at $10^{-7} \mathrm{M}$.

AChR was preincubated with the sera before addition of ${ }^{125} \mathrm{I}-$ $\alpha-B u T x$, all five AMC-M sera showed strong inhibition of ${ }^{125} \mathrm{I}-$ $\alpha-$ BuTx binding, with $40-60 \%$ inhibition at $4 \mu \mathrm{l}$ (Fig. $1 \mathrm{~B}$; Table II). Because the AChR in this assay was from denervated human muscle that expresses mainly fetal AChR (see Methods), these results suggested that each of the AMC-M sera contained antibodies that bind to the ${ }^{125} \mathrm{I}-\alpha-\mathrm{BuTx}$ site(s) on fetal-type AChR.

Functional effects on fetal-type AChR. To determine whether the AMC-M sera could inhibit fetal AChR function, we

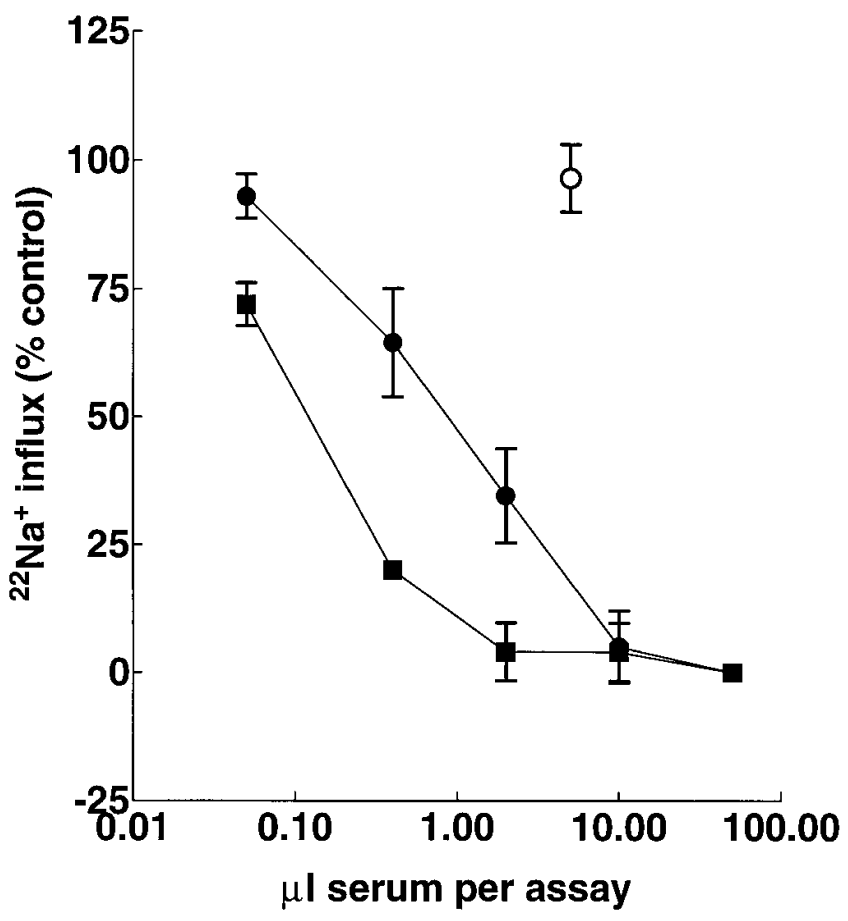

Figure 2. Inhibition of carbachol-induced ${ }^{22} \mathrm{Na}^{+}$flux into TE671- $\gamma$ cells (that express fetal-type AChR) by AMC-M sera. AMC-M1 (ם) and AMC-M2 (O) markedly inhibit flux compared with mean results ( \pm SD) from 10 healthy control sera at 1:100 dilution. The total volume in each assay was $500 \mu$ l. Results expressed as \% (mean \pm SD, $n=3$ ) of control wells in Hepes Locke solution only.


Figure 3. Inhibition of ACh-induced (bars) currents recorded at 10min intervals from Xenopus oocytes before and after a 40-min application of AMC-M2 IgG at 1:50 dilution (filled section). The IgG inhibits ACh-induced currents in oocytes expressing fetal-type AChR $\left(\alpha_{2} \beta \gamma \delta\right)$ but not in those expressing adult type $\left(\alpha_{2} \beta \delta \epsilon\right)$. The scale bar represents $250 \mathrm{nA}$ for fetal AChR and $125 \mathrm{nA}$ for adult AChR. Similar results were previously reported with IgG from AMC-M1 (8).

investigated their effect (1:100 dilution) on agonist-induced ${ }^{22} \mathrm{Na}^{+}$flux. In each case, AMC-M sera inhibited the ${ }^{22} \mathrm{Na}^{+}$flux into TE671- $\gamma$ cells (that express only fetal-type AChR) by $>85 \%$ (Table II), as shown in Fig. 2 for AMC-M1 and AMCM2. 10 healthy control sera at the same dilution had no effect compared with cells treated with medium alone.

To see whether the inhibitory effects of AMC sera were confined to fetal AChR, we tested their effects on TE671- $\epsilon$ cells that express predominantly adult AChR (15). None of the AMC-M sera, at 1:100 or 1:25 dilution, reduced AChR function in TE671- $\epsilon$ cells by more than $20 \%$ (Table II).

IgG from AMC-M1 and AMC-M2 was purified by affinity chromatography. The IgG fractions contained more than $90 \%$ of the anti-AChR antibody and of the activity that inhibited ${ }^{22} \mathrm{Na}^{+}$flux (not shown). AMC-M2 IgG at 1:50 dilution markedly reduced ACh-induced currents in Xenopus oocytes that had been injected with cRNA for the subunits of human fetal $\operatorname{AChR}\left(\alpha_{2} \beta \gamma \delta\right)$, but had no effect on Xenopus oocytes injected with the subunits for human adult AChR $\left(\alpha_{2} \beta \delta \epsilon\right)$ (Fig. 3).

Competition with anti-AChR monoclonal antibodies. The specificity of the sera for determinants on fetal-type AChR was further confirmed by the ability of fetal-specific mAbs to protect the AChR from the inhibitory actions that AMC-M1 and AMC-M2 sera exerted on ${ }^{22} \mathrm{Na}^{+}$flux. Three anti-AChR mAbs (B8, C2, and C9) that are fetal AChR-specific and bind to the $\gamma$ subunit (L. Jacobson, A. Vincent, and D. Beeson. 1996. In preparation), protected the AChR from subsequent 


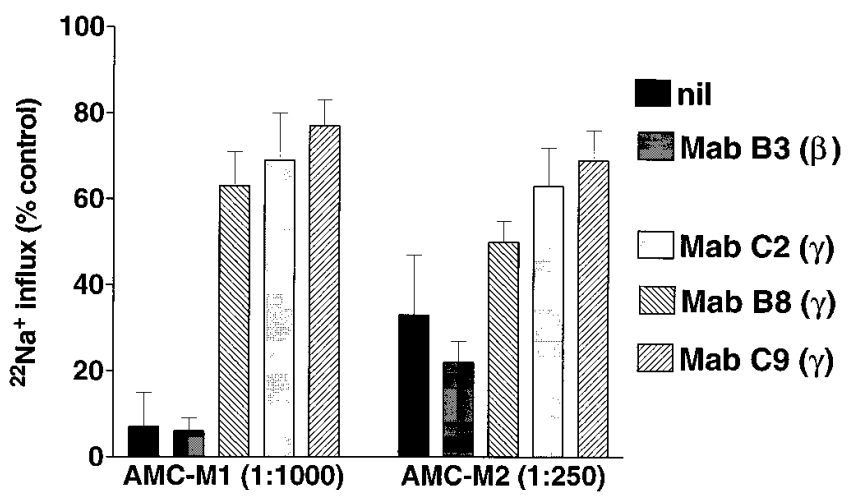

Figure 4. Protection of carbachol-induced ${ }^{22} \mathrm{Na}^{+}$flux in TE671- $\gamma$ from inhibition by AMC-M1 and AMC-M2 serum by mAbs to fetalAChR. TE671 cells were preincubated with the mAbs (1:250-1:500) before washing and addition of AMC-M1 (1:1000) or AMC-M2 (1:250) for $30 \mathrm{~min} .{ }^{22} \mathrm{Na}^{+}$flux results were expressed as \% (mean $\pm \mathrm{SD}, n=3$ ) of Hepes Locke treated controls.

The subunit specificity of the mAbs is indicated in the legends.

inhibition by AMC-M1 and AMC-M2 (Fig. 4). By contrast, $\mathrm{mAb} B 3$ that binds to the $\beta$ subunit on fetal and adult AChR (Jacobson, 1996) did not protect AChR function.

Anti-AChR in serum from unselected AMC mothers. AMC-M1 and AMC-M5 had no symptoms or signs of MG, in spite of raised levels of anti-AChR. We therefore assayed 20 sera from further mothers who had an obstetric history of AMC of unknown cause. The sera were negative for antiAChR antibodies by immunoprecipitation and did not inhibit ${ }^{125} \mathrm{I}-\alpha-\mathrm{BuTx}$ binding to fetal-type AChR (not shown). However, three of these sera inhibited fetal AChR function in the ${ }^{22} \mathrm{Na}^{+}$flux assay by more than $20 \%$, suggesting that there could

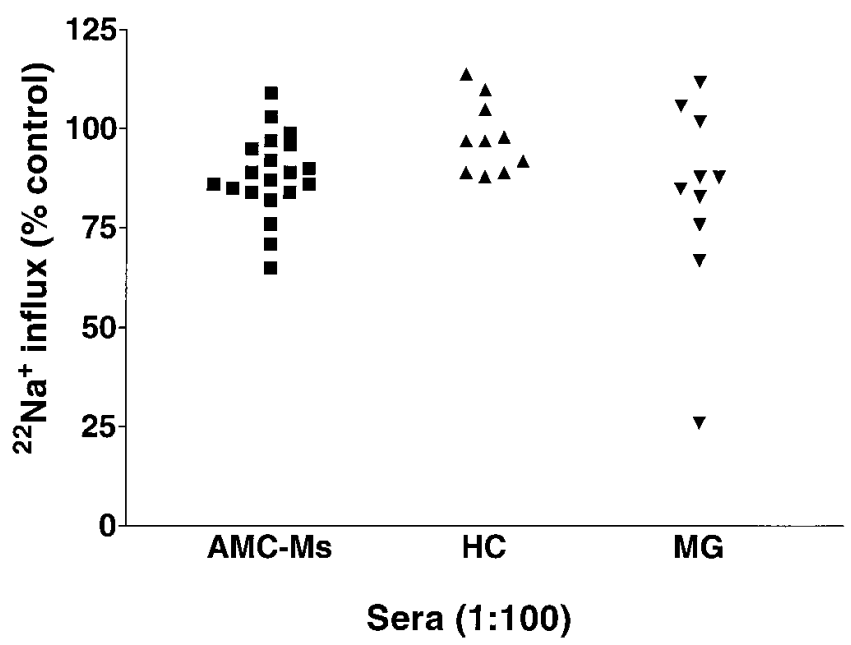

Figure 5. Inhibition of AChR function in TE671- $\gamma$ cells by sera from mothers with obstetric histories of AMC (AMC-Ms) without antiAChR antibodies. Results are compared with sera from typical MG patients (MG) and from healthy young controls (HC) on carbacholinduced ${ }^{22} \mathrm{Na}^{+}$flux in TE671- $\gamma$ cells. Wells were preincubated with sera at 1:100 dilution. Three AMC-M results $(74,71$, and $65 \%)$ were significantly different $(P<0.002$, Student's $t$ test; mean of three to eight determinations) from the mean $(98 \pm 9, n=10)$ of the healthy control results. be serum antibodies or other factors involved in these cases. Only 1 of 10 anti-AChR positive MG sera inhibited more strongly (Fig. 5).

\section{Discussion}

We have characterized the serum anti-AChR antibodies from five mothers whose obstetric histories had been complicated by AMC. In AMC-M1 and AMC-M2, recurrent severe intrauterine paralysis was responsible for stillbirths or termination of pregnancy $(8,9)$. In AMC-M3-5 the babies had both AMC and neonatal MG but survived with treatment (7). Neither AMC-M1 nor AMC-M5 showed symptoms of MG (7). Despite these differing clinical presentations, sera from all five women inhibited fetal AChR function but had little effect on the function of adult AChR. Some inhibition of fetal AChR function was also seen in three of 20 sera from other mothers with histories of fetal AMC. Thus, serum factor(s) that interfere with the function of AChR in the developing fetus may, although occurring rarely, be an important and potentially treatable cause of AMC.

IgG purified from AMC-M1 and AMC-M2 inhibited the ACh-induced currents of fetal, but not adult, AChR expressed in Xenopus oocytes (Fig. 3 and reference 8). ACh binds at the interfaces between the two $\alpha$ subunits and their adjacent subunits. These sites differ in antigenicity and affinity for ${ }^{125} \mathrm{I}-\alpha-$ BuTx on Torpedo AChR (18). Both fetal and adult forms of $\mathrm{AChR}$ have one $\alpha / \delta$ site; the second site in fetal AChR is formed by $\alpha / \gamma$, and in adult AChR by $\alpha / \epsilon$ (19). It is possible that the AMC-M antibodies inhibit fetal AChR function by binding to the $\alpha / \gamma \mathrm{ACh}$ binding site which is unique to fetal AChR. The fact that AMC-M sera also strongly inhibited approximately half the ${ }^{125} \mathrm{I}-\alpha-\mathrm{BuTx}$ binding sites on AChR extracted from denervated human muscle (AMC-M1, M2, and M4; Fig. $1 B$ ), and on surface AChR in TE671- $\gamma$ cells (AMC-M1 and AMC-M2, data not shown), is consistent with this hypothesis. Previous reports have found a relationship between inhibition of AChR function and inhibition of ${ }^{125} \mathrm{I}-\alpha-\mathrm{BuTx}$ binding with some MG sera $(20,21)$. Although AMC is found in only about $10 \%$ of babies with neonatal $\mathrm{MG}(7,22)$, the inhibition by serum of ${ }^{125} \mathrm{I}-\alpha-\mathrm{BuTx}$ binding to fetal/denervated AChR might be helpful in identifying mothers whose fetuses are at risk.

Preference of MG serum antibodies for fetal/denervated AChR has been demonstrated in previous studies (e.g. 7, 20, 21, 23, 24). Vernet-der Garabedian et al. (7) recently showed that mothers who transmit neonatal MG to their babies have a high proportion of antibodies that, in immunoprecipitation assays, bind to fetal AChR rather than to adult AChR. However, they did not find this preference in cases of neonatal MG with AMC (described as fetal damage in their paper), presumably because they did not look specifically for antibodies that inhibit ${ }^{125} \mathrm{I}-\alpha-$ BuTx binding to fetal AChR.

The results of this study raise several other questions. AMC-1 and AMC-5 had high anti-AChR levels by immunoprecipitation and yet did not have MG. Antibodies to AChR are usually considered diagnostic for $\mathrm{MG}$, and it is very rare to find high levels of anti-AChR antibody in individuals without any history of weakness (4). Anti-AChR antibodies in MG lead to loss of functional muscle (adult-type) AChR by a variable combination of complement-dependent lysis of the neuromuscular junction, accelerated degradation of AChR and di- 
rect block of AChR function (4). It would be interesting to clone IgG antibodies from mothers of AMC babies, using combinatorial expression libraries. One could then test the epitope specificity and pathogenicity of the individual Fabs or reconstructed IgGs.

Another question relates to the antigenic stimulus responsible for inducing the highly specific inhibitory antibodies found in AMC-Ms. Although fetal AChR is present in the normal thymus and some muscles $(25,26)$, it is possible that the antibodies in AMC occur as an immune response to fetal muscle during pregnancy. It will be interesting to compare the frequency of these antibodies in other MG patients, particularly between parous and nonparous women.

It has been suggested that the susceptibility of extraocular muscles to myasthenic weakness reflects the presence of fetal AChR on the multiply innervated tonic fibers (27). Although there is some expression of AChR $\gamma$ subunit mRNA in extraocular muscle (26), there is more $\mathrm{AChR} \epsilon$ subunit mRNA (MacLennan, C., D. Beeson, A. Vincent, J. Newsom-Davis, manuscript submitted for publication), and the lack of any ocular symptoms in AMC-M1 and AMC-M5 argues against antibodies to fetal AChR causing ocular muscle weakness.

The occurrence of high antibody titers against functional determinants on fetal AChR make it difficult to manage pregnancy in these cases. However, there must be increasing amounts of adult AChR made throughout the second and third trimesters since some mRNA for the $\epsilon$ subunit is expressed by 14 weeks (MacLennan, C., D. Beeson, A. Vincent, J. Newsom-Davis, in press) and most of the fetal form is lost by 33 wk gestation (28). Therefore treatment might be required for a limited period during midpregnancy only. Plasma exchange and intravenous immunoglobulin infusions might reduce the level of circulating antibodies to an extent that is compatible with fetal movement. Furthermore, the ability of mouse monoclonal antibodies to prevent binding of AMC-M1 and -M2 antibodies suggests the possibility of blocking the binding by monoclonal Fab fragments, or by peptides representing the fetal-specific functional epitope on the AChR. Alternatively, it may be possible to block the active Fc-mediated transport of maternal antibody across the placenta for a limited period of time during which the fetus is most at risk.

The causes of AMC are multifactorial: some are clearly genetic, but others are unknown. Environmental factors, viral infections, and maternal neurological or muscle disease associated with reduced fetal movements, have been implicated (1). When there is a recurrence in the absence of maternal disease, the etiology is usually presumed to be an autosomal recessive gene. We have shown that maternal antibodies are the cause in some cases. In addition to the five cases characterized here, we have screened sera from 20 mothers with an obstetric history of AMC; none were positive for anti-AChR but three of the sera inhibited AChR function at 1:100 dilution, albeit by a modest amount. Cases of neonatal MG with undetectable antiAChR have been reported, and seronegative MG appears to be due to antibodies binding to other muscle determinants that indirectly affect AChR function (29). It is possible that maternal antibodies directed at other determinants on fetal muscle may cause muscle weakness or damage in some anti-AChR negative AMC cases. Alternatively, circulating non-Ig inhibitory factors may be present.

The role of maternally derived antibodies in fetal damage is not limited to muscle. In particular, anti-Ro antibodies, from both symptomatic and asymptomatic mothers, can cause congenital heart block (30). Placental transfer of human antinuclear antigen antibodies has been demonstrated in mice injected with IgG from a patient with systemic lupus erythematosus (31). This mouse model should be employed to investigate the possible role of antibodies to other fetal specific antigens, or to neuronal antigens that are exposed during fetal development, in causing fetal death or congenital abnormalities.

\section{Acknowledgments}

We thank the Myasthenia Gravis Association/Muscular Dystrophy Group and the Medical Research Council of Great Britain for support. S. Riemersma was assisted by Biomed 1 project PL93 1100. We are very grateful to Mr. Leslie Jacobson, Dr. Nick Willcox, and Mr. Paul Plested for their help, and to Drs. Susan Huson, Louise Brueton, Jane Hurst, and other clinical geneticists who provided serum samples from their cases.

\section{References}

1. Hall, J.G., S.D. Reed, and G. Greene. 1982. The distal arthrogryposes: delineation of new entities-review and nosologic discussion. Am. J. Med. Genet. 11:185-239.

2. Drachman, D.B., and L. Sokoloff. 1966. The role of movement in embryonic joint development. Rev. Biol. 14:401-420.

3. Jago, R.N. 1970. Arthrogryposis following treatment of maternal tetanus with muscle relaxants. Arch. Dis. Childhood. 45:277-279.

4. Drachman, D.B. 1994. Myasthenia gravis. N. Engl. J. Med. 330:17971810.

5. Claudio, T. 1989. Molecular genetics of acetylcholine receptor-channels. In Frontiers in Molecular Neurobiology. D.M. Glover, and B.D. Hames, editors. IRL Press, Oxford. 63-142.

6. Morel, E., B. Eymard, B. Vernet-der Garabedian, C. Pannier, O. Dulac, and J.-F. Bach. 1988. Neonatal myasthenia gravis: a new clinical and immunologic appraisal on 30 cases. Neurology. 38:138-142.

7. Vernet-der Garabedian, B., M. Lacokova, B. Eymard, E. Morel, M. Faltin, J. Zajac, O. Sadovsky, M. Dommergues, P. Tripon, and J.F. Bach. 1994. Association of neonatal myasthenia gravis with antibodies against the fetal acetylcholine receptor. J. Clin. Invest. 94:555-559.

8. Vincent, A., C. Newland, L. Brueton, D. Beeson, S. Riemersma, S.M. Huson, and J. Newsom-Davis. 1995. Arthrogryposis multiplex congenita with maternal autoantibodies specific for a fetal antigen. Lancet. 346:24-25.

9. Barnes, P.R.J., D.J. Kanabar, L. Brueton, J. Newsom-Davis, S.M. Huson, N.P. Mann, and D. Hilton-Jones. 1995. Recurrent congenital arthrogryposis leading to a diagnosis of myasthenia gravis in an initially asymptomatic mother. Neuromusc. Dis. 5:59-65.

10. Vincent, A., S. Riemersma, D. Beeson, S. Huson, L. Brueton, C. Newland, and J. Newsom-Davis. 1996. Arthrogryposis multiplex congenita associated with maternal antibodies to fetal acetylcholine receptor. Neurology. 46: A112.

11. Vincent, A., and J. Newsom-Davis. 1985. Acetylcholine receptor antibody as a diagnostic test for myasthenia gravis: results in 153 validated cases and 2967 diagnostic assays. J. Neurol. Neurosurg. Psychiatry. 48:1246-1252.

12. Whiting, P.J., A. Vincent, M. Schluep, and J. Newsom-Davis. 1986. Monoclonal antibodies that distinguish between normal and denervated human acetylcholine receptor. J. Neuroimmunol. 11:223-235.

13. Lang, B., G. Richardson, J. Rees, A. Vincent, and J. Newsom-Davis. 1988. Plasma from myasthenia gravis patients reduces acetylcholine receptor agonist-induced $\mathrm{Na}+$ flux into TE671 cell line. J. Neuroimmunol. 19:141-148.

14. Yamamoto, T., A. Vincent, T.A. Ciulla, B. Lang, I. Johnston, and J. Newsom-Davis. 1991. Seronegative myasthenia gravis: A plasma factor inhibiting agonist-induced acetylcholine receptor function copurifies with IgM. Ann. Neurol. 30:550-557.

15. Beeson, D., M. Amar, I. Bermudez, A. Vincent, and J. Newsom-Davis. 1996. Stable functional expression of the adult subtype of human muscle acetylcholine receptor following transfection of the human rhabdomyosarcoma cell line TE671 with cDNA encoding the $\epsilon$ subunit. Neurosci. Lett. 207:57-60.

16. Newland, C.F., D. Beeson, A. Vincent, and J. Newsom-Davis. 1995. Functional and non-functional isoforms of the human muscle acetylcholine receptor. J. Physiol. (Lond.). 489:767-778.

17. Lang, B., A. Vincent, and J. Newsom-Davis. (1982). Purification of antiacetylcholine receptor antibody from patients with myasthenia gravis. J. Immunol. Meth. 51:371-381.

18. Dowding, A.J., and Z.H. Hall. 1987. Monoclonal antibodies specific for each of the two toxin-binding sites of Torpedo acetylcholine receptor. Bio- 
chemistry. 26:6372-6381.

19. Karlin, A., and M.H. Akabas. 1995. Toward a Structural Basis for the Function of Nicotinic Acetylcholine Receptors and Their Cousins. Neuron. 15: 1231-1244.

20. Hall, Z.W., S. Pizzighella, Y. Gu, S. Vicini, and S.M. Schuetze. 1987. Functional inhibition of acetylcholine receptors by antibodies in myasthenic sera. Ann. NY Acad. Sci. 505:272-285.

21. Burges, J., D.W. Wray, S. Pizzighella, Z. Hall, and A. Vincent. 1990. A myasthenia gravis plasma immunoglobulin reduces miniature endplate potentials at human endplates in vitro. Muscle Nerve. 13:407-413.

22. Dinger, J., and B. Prager. 1993. Arthrogryposis multiplex in a newborn of a myasthenic mother-case report and literature. Neuromusc. Dis. 3:335339 .

23. Weinberg, C.B., and Z.W. Hall. 1979. Antibodies from patients with myasthenia gravis recognize determinants unique to extrajunctional acetylcholine receptors. Proc. Natl. Acad. Sci. USA. 76:504-508.

24. Vincent, A., and J. Newsom-Davis. 1982. Acetylcholine receptor antibody characteristics in myasthenia gravis. I. Patients with generalized myasthenia or disease restricted to ocular muscles. Clin. Exp. Immunol. 49:257-265.
25. Schluep, M., N. Willcox, A. Vincent, G.K. Dhoot, and J. NewsomDavis. 1987. Acetylcholine receptors in human thymic myoid cells in situ: an immunohistological study. Ann. Neurol. 22:212-222.

26. Horton, R.M., A.A. Manfredi, and B-M. Conti-Tronconi. 1993. The "embryonic" gamma subunit of the nicotinic acetylcholine receptor is expressed in adult extraocular muscle. Neurology. 43:983-985.

27. Kaminski, H.J., E. Maas, P. Spiegel, and R.L. Ruff. 1990. Why are eye muscles frequently involved in myasthenia gravis? Neurology. 40:1663-1669.

28. Hesselmans, L., F. Jennekens, C. van den Oord, H. Veldman, and A. Vincent. 1993. Immunoreactivity to the acetylcholine receptor in developing human muscle. Anat. Rec. 236:553-562.

29. Barrett-Jolley, R., A. Vincent, N. Byrne, and J. Newsom-Davis. (1994). Plasma from patients with seronegative myasthenia gravis inhibit nAChR responses in the TE671/RD cell line. Pflueg. Arch. Euro. 428:492-498.

30. Olson, N.Y., and C.B. Lindsley. 1987. Neonatal lupus syndrome. Am. J. Dis. Child. 141:908-910.

31. Guzman-Enriquez, L., E. Avalos-Diaz, and R. Herrera-Esparza. 1990. Transplacental transfer of human antinuclear antibodies in mice by injection of lupus IgG in pregnant animals. J. Rheumatol. 17:52-56. 